\title{
Vouloir citationnel. Chronique du changement métonymique d'un verbe modal français
}

\author{
Stefanie Goldschmitt \& Andrea Landvogt \\ Julius-Maximilians-Universität Würzburg, Allemagne
}

\section{Introduction}

La 'valeur citationnelle' que le verbe modal pouvoir peut exprimer dans certaines circonstances est un phénomène jusque-là peu étudié. Il est vrai que les occurrences sont rares, mais en revanche, on les trouve déjà à partir du $\mathrm{XIV}^{\mathrm{e}}$ siècle. Dans cette acception qui est issue d'un changement sémantique, vouloir tend à perdre sa signification d'origine pour adopter une fonction nouvelle. Au lieu d'exprimer une valeur volitive, il signale désormais les réserves qu'un locuteur-rapporteur prend vis-à-vis des paroles et des points de vue attribués à un autre locuteur. Avant d'illustrer l'acception citationnelle de vouloir, il convient néanmoins de montrer qu'elle n'est pas l'unique écart dans la signification principale de ce verbe, c'est-à-dire la valeur volitive. Bien au contraire, vouloir est un verbe polysémique - fait avéré déjà dans l'antiquité.

\section{Vouloir - verbe polysémique}

Étymologiquement, le verbe vouloir remonte à VOLERE en latin vulgaire provenant de la forme VELLE du latin classique. Dans l'histoire de la langue, le noyau sémantique de ce verbe - la volition - est resté relativement stable. Ainsi, la forte majorité des occurrences a une signification volitive, en latin et en français :

Idem velle atque idem nolle, ea demum firma amicitia est.

'Vouloir les mêmes choses et détester les mêmes choses, c'est ce qui rend l'amitié stable.'

Elle veut ressembler à Jeanne d'Arc, dans ses haillons de fille maudite. (Express, 68)

'Elle désire ressembler à Jeanne d'Arc (...)'

Depuis le latin classique, le verbe vouloir a connu une deuxième valeur : la signification de futur qui, de nos jours, se trouve encore dans certains dialectes de l'est de la France et en Suisse. ${ }^{1}$ Déjà existante en latin classique (3), cette signification s'est conservée à travers les siècles suivants (4) et (5) :

Volo tibi commemorare, si forte eadem res tibi dolorem minuere possit. (Cic. ep. ad fam., 4, 5,4 )

'Je te le rappellerai si la même chose pourra diminuer ta douleur.' ${ }^{2}$

Scio te, cum ista legeris, meum os digito velle comprimere. (Hier. ep., 27, 2)

'Je sais que tu voudras/vas fermer ma bouche avec ton doigt quand tu liras ceci.'

Si admittimus hostes, jam properare volent. (Joh. 6, 250 ; de Corippus, $\mathrm{VI}^{\mathrm{e}}$ s. après J.-C.)

'Si nous admettons des ennemis, ils voudront/vont se dépêcher.'

La valeur de futur persiste également en français $:^{3}$

$$
\begin{aligned}
& \text { Ce mur veut tomber. (TLF, s.v. vouloir) } \\
& \text { 'Ce mur va (bientôt) tomber.' }
\end{aligned}
$$

Contrairement au latin, le verbe connaît une troisième signification en français : à l'emploi volitif et futur de vouloir s'ajoute l'emploi citationnel dont nous nous occuperons dans la présente étude. 
Or, tout d'abord, il faut discerner deux formes du verbe vouloir qui diffèrent du point de vue syntaxique : le verbe au sémantisme plein et l'auxiliaire modal. ${ }^{4}$ Le verbe plein traduit généralement un désir du sujet. Toutefois, il existe des constructions où on ne peut pas discerner s'il s'agit d'un désir ou d'une intention.

Quant au verbe modal, il peut exprimer deux types de volition, soit un désir, soit une intention du sujet. Les exemples suivants permettent d'illustrer les nuances volitives en question : (7) comporte un exemple de l'emploi volitif du verbe plein. (8) illustre la construction avec un objet prédicatif qui, selon nous est ambiguë, puisqu'elle peut exprimer soit la volition soit l'intention de la part de l'agent.

(7) Je mesurai sa force : elle avait voulu mon père, elle l'avait, elle allait peu à peu faire de nous le mari et la belle-fille d'Anne Larsen. (Sagan, 77)

'(...) elle avait désiré (posséder) mon père (...)'

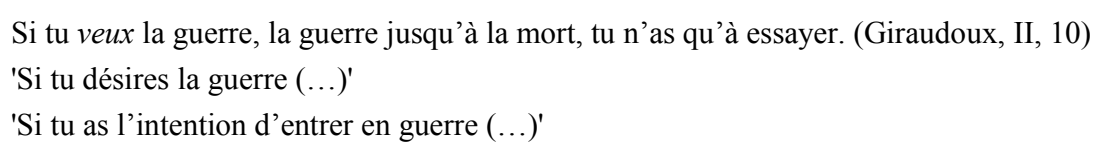

Dans les exemples suivants, vouloir figure en tant que verbe modal. Ainsi, en (9), il exprime le désir et en (10) il traduit l'intention de l'agent de la phrase; or, dans chacun des deux cas, les significations alternatives - intention pour (9) et désir pour (10) - ne sont pas exclues.

(9) Je veux être un roi sans terre et sans sujets. (Sartre, III, 6)

'J'ai envie d'être un roi (...)'

'J'ai l'intention d'être un roi (...)'

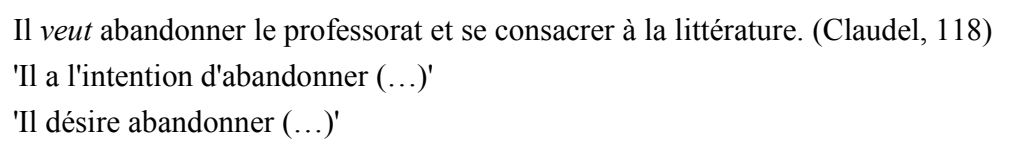

Du point de vue sémantique, les deux interprétations du modal vouloir ne se distinguent pas toujours nettement. Dépendant du contexte, le désir et l'intention ne sont pas des significations autonomes. En effet, les exemples (9) et (10) représentent des cas de transition dans lesquels les deux valeurs - désir et intention - se superposent au point où il n'est plus possible de les distinguer sans précisions contextuelles ultérieures.

Par la suite, nous considérerons uniquement les emplois de vouloir en tant qu'auxiliaire. Plus précisément, nous écarterons la valeur future et nous nous limiterons à l'analyse de deux usages de l'auxiliaire modal vouloir $:{ }^{5}$ l'emploi volitif et l'emploi citationnel. ${ }^{6}$ Afin de décrire les différentes valeurs, nous nous appuyons sur une approche développée par Heine (1995) pour décrire le changement sémantique de l'interprétation déontique à l'interprétation épistémique subi par les verbes modaux allemands.

\section{L'emploi volitif du verbe modal vouloir}

Selon Heine (1995), l'interprétation déontique dépend de cinq caractéristiques conceptuelles (conceptual properties) : ?

- $\quad$ une force (F) qui est caractérisée par un élément de volonté (force)

- $\quad$ un agent de contrôle (C) qui est responsable de l'acte (controlling agent)

- $\quad$ un événement dynamique (D) (dynamic)

- $\quad$ la postériorité de l'événement par rapport au temps de référence (L) (later than the reference time)

- $\quad$ le degré de probabilité $(\mathrm{P})$ indiquant l'éventuelle réalisation de l'événement (probability). 
Selon nous, ces cinq critères sont également valables pour l'interprétation volitive de vouloir. Leur pertinence sera démontrée à l'aide d'un exemple que nous avons déjà mentionné plus haut :

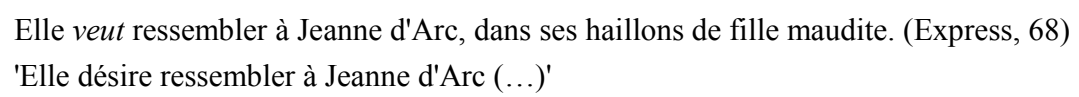

Le premier facteur, la force $(\mathrm{F})$, consiste en la volonté de l'agent : la jeune fille désire ressembler à Jeanne d'Arc. Cette force implique un élément de volonté dans la mesure où la fille en question a un intérêt personnel dans la réalisation de l'événement voire dans sa non-réalisation.

Le deuxième facteur $(\mathrm{C})$ se réfère à l'agent sur lequel la force $(\mathrm{F})$ est efficace; dans notre exemple l'agent de contrôle serait Jeanne d'Arc. Vu que, dans le cas du verbe vouloir, la force coïncide avec l'agent (C), il s'agit d'une force intra-subjective. ${ }^{8}$

Quant au troisième facteur (D), il a moins d'importance pour la valeur volitive que les précédents puisque les emplois déontiques sont plus souvent associés aux événements dynamiques qu'aux infinitifs statiques. Notre exemple montre que ce critère est négligeable pour l'interprétation volitive de vouloir, car ce verbe modal se combine facilement avec des infinitifs ; dynamiques - p.ex. abandonner dans (10) - et statiques - p.ex. ressembler dans (2).

Le facteur (L), par contre, constitue une caractéristique définitoire. Généralement, les événements régis par vouloir se déroulent dans l'avenir ou, moins souvent, dans le présent. En revanche, les références au passé sont moins courantes quand il s'agit d'une modalité volitive.

Le cinquième facteur $(\mathrm{P})$ - qui s'avérera d'ailleurs particulièrement important pour notre problématique est un trait caractéristique du volitif en général $:^{9}$ L'événement ne représente pas un fait qui existe de manière objective, mais la probabilité concerne le désir de la fille de voir se réaliser son souhait. Le degré de probabilité dépend de la force $(\mathrm{F})$. Si ses efforts sont suffisamment grands, la jeune fille réussira probablement à ressembler à Jeanne d'Arc. Il s'avère que le degré de probabilité $(\mathrm{P})$ est étroitement associé à la force $(\mathrm{F})$ : quand le degré de la force $(\mathrm{F})$ est élevé, le degré de la probabilité $(\mathrm{P})$ l'est également.

\section{L'emploi citationnel du verbe modal vouloir}

À part sa valeur volitive, le modal vouloir a développé un emploi ultérieur qui est souvent négligé dans la recherche à cause de sa rareté : l'emploi citationnel. Cette rareté est fort probablement liée aux restrictions de l'emploi citationnel de vouloir.

\subsection{La prédilection pour la troisième personne}

Notre première hypothèse est que cet emploi privilégie clairement la troisième personne. Il est vrai que la première personne ne s'y prête pas trop pour des raisons d'argumentation : en général, l'autocitation n'est pas utilisée pour se caractériser soi-même comme locuteur hypocrite. Ceci n'est pas étonnant dans la mesure où nous ne sommes confrontés à un dédoublement des instances énonciatives : le locuteur - le 'je' - coïncide avec l'agent de contrôle (C). Ainsi, les exemples montrent que la lecture volitive est prédominante à la première personne (au singulier aussi bien qu'au pluriel). Or, on peut se demander si un tel emploi serait, du moins théoriquement, possible (par exemple: Tu es sûr que je voulais avoir fait les courses? J'ai vraiment dit cela?) Toutefois, nous n'avons pas trouvé d'exemple attestant cet emploi.

Quant aux emplois avec la deuxième personne, nous n'avons pas non plus relevé d'exemples, ni dans Frantext ni sur internet. L'emploi à la deuxième personne présupposerait une agression pour l'interlocuteur; elle ne paraît probable que dans des questions moqueuses. Malgré ce résultat (intermédiaire), un tel emploi citationnel ne peut pas être définitivement exclu (par exemple : Et toi, tu veux avoir réussi à l'examen? C'est ridicule!). Bien que cette forme soit théoriquement possible, nos 
recherches n'ont pas fourni d'occurrences exigeant une interprétation citationnelle avec le verbe modal à la deuxième personne.

Il n'est donc point surprenant que les exemples que nous donnons soient tous des occurrences affichant la troisième personne.

\subsection{La construction 'vouloir + infinitif accompli'}

Nos recherches montrent que les occurrences de vouloir ayant (clairement) une interprétation citationnelle, sont de préférence suivies d'un infinitif accompli, ce dernier permettant de faire un résumé de ce qui a été dit. ${ }^{10}$ Dans la mesure où l'infinitif figure sous sa forme accomplie, il implique l'antériorité de l'énonciation rapportée. Grâce à cette antériorité, vouloir peut acquérir sa notion citationnelle. Le décalage temporel de l'énonciation citée par rapport à l'énonciation actuelle est interprété comme indicateur de la divergence des situations.

Regardons de près quelques exemples. Nous avons déjà dit que les exemples de l'emploi citationnel de vouloir ne sont pas nombreux. Toutefois, cet emploi mérite notre attention car, bien que rare en général, le premier exemple que nous avons relevé date déjà du XIV ${ }^{\mathrm{e}}$ siècle :

(11) Après advint que le roi Philippe emprit et accueillit ce messire Robert en si grand'haine, pour occasion d'un plaid qui ému étoit devant lui, dont le comte d'Artois étoit cause, que le dit messire Robert vouloit avoir gagné, par vertu d'une lettre que messire Robert mit avant, qui n'étoit mie bien vraie, si comme on disoit, que si le roi l'eût tenu en son ire il l'eût fait mourir sans nul remède. (Froissart 1972, 146)

'(...) que le dit monsieur Robert prétendait avoir gagné (...)'

Dans l'exemple précédent, la signification prédominante du verbe vouloir n'est plus la volition, mais le jugement du locuteur : il cite l'énoncé d'un premier locuteur. En même temps, le choix du modal vouloir indique le doute du rapporteur quant à la vérité des paroles rapportées. Ainsi, l'occurrence (11) exige une paraphrase avec prétendre.

Les exemples suivants demandent le même type de paraphrase :

(12) Maître Jean fit apporter du cidre, et longtemps autour de la table on parla de ce qui venait de se passer. Chacun voulait avoir dit son mot, ceux qui n'avaient pas soufflé, comme les autres, mais tous reconnaissaient le courage et le bon sens de Marguerite. (Erckmann \& Chatrian 1962, 19)

'(...) chacun prétendait avoir dit son mot (...)'

Cette occurrence est encore plus explicite : même ceux qui, à l'époque, n'avaient rien dit, prétendaient avoir parlé. Il ne s'agit donc que d'une prétention de la part des locuteurs cités par le narrateur. ${ }^{11}$

La lecture de l'exemple (13) exclut également une interprétation volitive ; à cause du contexte, seule l'interprétation citationnelle est acceptable :

(13) Les confessions en ce point, toutes ces pages sur son 'apostasie' nous rapportent la vie que Jean-Jacques voulait avoir vécue, bien plus que celle qu'il avait vécue en effet. (Guéhenno 1948, 47)

'(...) la vie que Jean-Jacques prétendait avoir vécue (...)'

Ici, le critique du livre fait un commentaire subjectif : il croit que les Confessions nous racontent la vie de Rousseau telle que ce dernier prétend l'avoir vécue et non pas la véritable histoire de sa vie. La proposition suivant ce commentaire - bien plus que celle [la vie] qu'il avait vécue en effet souligne que l'auteur doute de la vérité du premier énoncé.

Les occurrences (11), (12) et (13) ne permettent pas de lecture volitive du verbe modal. Mais il ne faut pas croire que tous les occurrences de 'vouloir + infinitif accompli à la troisième personne' suggèreraient l'interprétation citationnelle, au contraire. On trouve aussi des contre-exemples ayant gardé la valeur volitive. Dans les exemples (14) et (15), c'est clairement la valeur volitive qui persiste : 

du Gard, 409)

'(...) tous désirent avoir vu'

Les livres sont très chers, et tout cela y est pour quelque chose ; c'est bien la preuve que les gens se soucient peu d'acheter de la bonne littérature ; ils veulent avoir lu le livre recommandé par leur club, celui dont on parle, et ils se moquent bien de ce qu'il y a dedans.

'ils désirent avoir lu le livre (...')

D'autres exemples, en revanche, permettent toutes les deux lectures. En (16) une interprétation volitive nous semble aussi probable qu'une lecture citationnelle.

(16) - Bon! bon! dit Saccage au capitaine, il n'est rien de tel que de menacer! À présent, les voilà tous qui veulent avoir servi sous vos ordres. (Sand, 93)

'les voilà tous qui désirent avoir servi sous vos ordres'

'les voilà tous qui prétendent avoir servi sous vos ordres'

Dans ce passage, même le contexte ne fournit pas d'indices définitifs aidant à désambiguïser la signification de la phrase. Cette ambiguïté nous indique que les deux lectures sont très proches et que la transition sémantique de l'emploi volitif à l'emploi citationnel se passe 'en douce'.

\section{Le changement sémantique sous-jacent : métaphorique ou métonymique ?}

Quant à la signification de l'emploi citationnel de vouloir, elle peut être cernée comme suit. Puisqu'il s'agit du citationnel, nous avons affaire à deux niveaux énonciatifs : l'énonciation enchâssante actuelle et l'énonciation enchâssée, rapportée. ${ }^{12}$ En utilisant la formule 'vouloir + infinitif accompli', le locuteur actuel affirme qu'un deuxième locuteur a manifesté un certain point de vue, tout en affichant explicitement son propre doute vis-à-vis de ce point de vue d'autrui. On pourrait dire que le locuteurrapporteur reprend le discours cité à la manière d'un résumé. En même temps, il souligne qu'il entretient un lien énonciatif dissociatif avec l'énoncé cité, c'est-à-dire qu'il n'assume pas la responsabilité de ce qui a été dit par l'autre. ${ }^{13}$ Ce type d'emploi du modal vouloir fonctionne donc comme un marqueur de polyphonie discursive, respectivement comme un «discordanciel énonciatif» (Rosier 1998, 288), puisqu'il implique toujours au moins deux locuteurs. Dans la mesure où ces locuteurs sont à considérer comme étant des « sources » ${ }^{14}$ de deux points de vue contradictoires, on pourrait même dire qu'il s'agit d'un marqueur de « dialogicité » au sens de Bachtin $(1971,222)$.

À première vue, cette nouvelle signification citationnelle semble très éloignée de la signification primaire du verbe. Par la suite, nous essayerons de montrer comment elle a pu se développer et, dans un deuxième temps, nous tenterons de mieux cerner la nature de cette évolution sémantique. Nous partons du principe que le changement de l'emploi volitif au citationnel correspond grosso modo à celui de l'usage déontique des verbes modaux à l'usage épistémique.

En ce qui concerne ce changement, il existe deux positions dans la recherche: alors que certains postulent qu'il est de nature métaphorique, ${ }^{15}$ d'autres y voient un cas de métonymie. ${ }^{16}$ Le changement métaphorique implique un 'saut conceptuel' entre deux domaines différents :

The relation between deontic and epistemic modality can in fact be described as being metaphorical in nature, involving a transfer between two domains of conceptualization. The former implies a 'dynamic' world of willful human beings who act and are capable of imposing their will on other agents. The latter, on the other hand, essentially implies a 'static' world, one that may but need not be associated with human participants. (Heine, Claudi \& Hünnemeyer 1991, 175)

Un changement métonymique, en revanche, présuppose un lien de contiguïté entre les deux domaines en question. 
Il s'en suit que les critères pertinents pour décider de la nature du changement sémantique - la métaphore impliquant une non-contiguité versus la métonymie exigeant une contiguité - se distinguent. Nos recherches ont démontré qu'en ce qui concerne le verbe vouloir dans son emploi citationnel, il faut suivre la piste de la métonymie.

Prenons une occurrence ambiguë (17) pour montrer que la lecture volitive peut être très proche de la lecture citationnelle. Dans ce cas, l'ambiguité est favorisée par le fait que l'exemple (17) ne contient pas un infinitif accompli, mais un infinitif simple. Ceci facilite les deux lectures qui nous intéressent:

$$
\begin{aligned}
& \text { Si les fondamentalistes ont voulu me tuer, c'est parce qu'ils veulent appliquer le vrai islam. (Express, 104) } \\
& \text { '(...) ils ont l'intention de/désirent appliquer le vrai islam (...)' } \\
& \text { '(...) ils prétendent appliquer le vrai islam (...)' }
\end{aligned}
$$

Il s'agit d'un énoncé extrait d'une interview avec une jeune musulmane qui plaide contre une interprétation fondamentaliste de l'islam - erronée selon elle. Le contexte permet de déduire que la locutrice ne croit pas que les fondamentalistes aient bien compris l'islam. À son avis, les fondamentalistes ne font que prétendre appliquer le vrai islam. Si l'on y voit une occurrence citationnelle, cela implique que la jeune fille fait allusion à des énoncés préexistants du type Nous, on applique le vrai islam. En même temps, elle ne prend pas la responsabilité de ce point de vue rapporté ; au contraire, elle le rejette.

Comme la première des paraphrases le montre, on ne peut pas non plus dénier l'interprétation selon laquelle les islamistes seraient poussés par l'intention ou le désir d'appliquer le Coran comme il se doit. Dans ces deux lectures, c'est la victime qui fournit une explication pour le comportement des fondamentalistes. Elle ne se distancie pourtant pas clairement du motif qu'elle croît être à l'origine de l'agression contre elle. Dans ce cas, c'est le lecteur qui doit décider de l'interprétation à donner selon le contexte de l'énoncé.

Pour qu'une telle ambiguité soit possible, la lecture volitive doit être relativement proche de la lecture citationnelle. Elle présuppose une continuité conceptuelle entre les deux interprétations, une sorte de continuum sémantique. Cette idée d'un continuum suggère qu'il s'agit d'un changement métonymique plutôt que d'un changement métaphorique.

Afin de mieux comprendre le rapport entre les deux lectures, la relation contiguë doit être examinée en détail à l'aide des cinq caractéristiques susnommées des auxiliaires modaux. La pertinence de ces critères sera évaluée à partir d'un de nos exemples non ambigus :

(13) Les confessions en ce point, toutes ces pages sur son 'apostasie' nous rapportent la vie que Jean-Jacques voulait avoir vécue, bien plus que celle qu'il avait vécue en effet. (Guéhenno 1948, 47)

'(...) la vie que Jean-Jacques prétendait avoir vécue (...)'

Dans la lecture qu'indique la paraphrase, la valeur volitive du modal n'est pas au premier plan. Par conséquent, il n'y a ni force (F) ni agent (C) sur lequel cette force serait efficace. Comme mentionné plus haut, le facteur (D) - dynamic - n'a pas de pertinence non plus. De même, le facteur (L) - later than reference time - est sans importance étant donné que l'événement se réfère au passé.

En revanche, c'est le cinquième facteur $(\mathrm{P})$ - la probabilité - qui devient essentiel pour la signification citationnelle : selon le locuteur, le critique, il est peu probable que Jean-Jacques ait vraiment vécu la vie telle qu'il la présente dans ses Confessions. Au contraire, le critique littéraire estime que la vie narrée dans les Confessions est une fiction prétendue par l'écrivain. Dans la seconde partie de la phrase, le critique souligne d'ailleurs son avis en affirmant que cette vie était « en effet » différente.

La particularité de l'emploi citationnel de vouloir consiste en le fait que le locuteur citant nomme toujours le locuteur originel. Par conséquent, l'agent de contrôle (C) de la proposition citée est repris dans l'énoncé actuel, mais il y est représenté de manière 'indirecte'. Le facteur (C) persiste donc de façon 'atténuée', tout comme le facteur (F), inhérent à l'agent (C). 
Ainsi, la probabilité (P) s'impose comme facteur principal pendant que les facteurs (C) et (F) sont nettement affaiblis, tout en étant potentiellement perceptibles. Voilà pourquoi on les dénotera comme suit : ${ }^{(\mathrm{C})} \mathrm{et}^{(\mathrm{F})}$. En revanche, les deux autres facteurs, (D) et $(\mathrm{L})$, reculent complètement à l'arrière-plan.

Ce fait devient plus clair quand on se rend compte que le locuteur actuel ${ }^{17}$ dans (12) cite un autre locuteur, Jean-Jacques. Le dernier est l'agent originel affecté par la force dans un énoncé volitif présupposé exprimant son désir. La force est donc, nous l'avons dit, intra-subjective. Ainsi, dans l'énoncé présupposé - Je veux vivre une telle vie - les facteurs $(\mathrm{F})$ et $(\mathrm{C})$ coïncident, la force $(\mathrm{F})$ étant efficace sur l'agent (C), c'est-à-dire sur Jean-Jacques.

Dans (13), le locuteur citant se réfère à l'énoncé volitif et, de façon indirecte, à l'agent (C), Jean-Jacques, en sa qualité de locuteur cité. Or, il y a une divergence entre le sujet qui parle et le sujet qui figure comme agent de contrôle. De cette divergence naît l'impression de prise de distance par rapport au discours cité qui est caractéristique de l'emploi citationnel de vouloir.

Par la suite, nous aimerions résumer ces transformations sémantiques dans un schéma afin de pouvoir décider du type de changement sémantique : s'agit-il d'une métaphore ou bien d'une métonymie ?

Le modèle figure/fond ${ }^{18}$ avec son premier plan (= figure) et son arrière plan (= fond) se prête à l'explication du phénomène décrit. La figure dans notre schéma renvoie à la lecture dominante du verbe vouloir dans son contexte ; le fond représente la possibilité d'une autre lecture dans l'arrière-plan :

\begin{tabular}{|c|c|c|c|}
\hline \multicolumn{2}{|c|}{ signification } & \multicolumn{2}{c|}{ contiguïté conceptuelle } \\
\hline & vouloir & emploi volitif & emploi citationnel \\
\hline emploi volitif & (F) (C) (D) (L) (P) & figure & fond \\
\hline emploi citationnel & (P) $($ F) (C) & fond & figure \\
\hline
\end{tabular}

Tableau 1 : Modèle figure/fond de l'emploi citationnel de vouloir

Pour l'interprétation volitive, les cinq facteurs (F), (C), (D), (L) et (P) se retrouvent au premier plan et constituent la figure dans le modèle - c'est-à-dire qu'ils sont typiques, mais non contraignants. Dès que les cinq facteurs sont saillants dans un énoncé, il s'agit d'une valeur volitive.

Quant à l'interprétation citationnelle, un seul facteur - la probabilité $(\mathrm{P})$ - passe au premier plan et devient la (nouvelle) figure. Les autres facteurs sont soit repoussés à l'arrière-plan où ils ne constituent désormais que le fond de la valeur épistémique, ou bien ils sont transformés dans la mesure où ils affectent le degré de la probabilité. Dans notre schéma, cette influence sur le facteur $(\mathrm{P})$ est symbolisée par les lettres $(\mathrm{F})$ et $(\mathrm{C})$ placées en hauteur.

Nous avons vu que la probabilité (P) devient la caractéristique centrale de la valeur citationnelle de vouloir. Or, il ne s'agit pas d'une composante de signification entièrement nouvelle ; elle préfigurait déjà en tant que facteur de fond de l'interprétation volitive. Dans la mesure où les autres facteurs disparaissent, elle acquiert un nouveau poids significatif.

Le facteur $(\mathrm{P})$ est donc la constante qui établit la relation de contiguité entre les deux interprétations possibles. Il n'y a pas de 'saut' entre les deux valeurs - volitive vs. citationnelle - comme l'exigerait le changement métaphorique, au contraire: puisque les deux emplois en question partagent un trait commun, il nous paraît justifié de parler d'un changement métonymique. Comme nous l'avons vu plus haut, les deux interprétations sont proches, les exemples s'avérant parfois même occurrences ambiguës. Cette proximité sémantique est un autre argument en faveur de notre position favorisant la métonymie. 
Vu qu'il ne subsiste qu'un facteur de fond du faisceau des cinq traits sémantiques de la signification principale, on pourrait avancer que la valeur citationnelle de vouloir représente une espèce de 'sortie de la voie principale' de la sémantique de ce verbe.

\section{Conclusion}

Dans notre proposition de communication, nous avons concentré notre analyse sur un emploi plutôt exotique du verbe modal vouloir : sa valeur citationnelle qui marque une polyphonie discursive, voire une dialogicité entre deux énoncés. D'après l'analyse de quelques occurrences exemplaires, cet usage paraît être associé à la combinaison d'un emploi de vouloir à la troisième personne et d'un infinitif accompli.

Nous avons essayé de corroborer notre hypothèse à l'aide d'un corpus plus vaste, apte à démontrer l'évolution diachronique de cette forme. Il restait à vérifier si l'on pouvait trouver des cas dans lesquels la première ou la deuxième personne entreraient en jeu. Or, dans nos recherches, nous avons vu que les emplois avec la première personne sont exclusivement de nature volitive, c'est-à-dire qu'ils expriment soit le désir soit l'intention du locuteur. Quant aux emplois à la deuxième personne, il n'a pas été possible de relever des exemples, ni dans Frantext ni sur internet. Il s'avère donc que le choix de la troisième personne est impératif pour une interprétation citationnelle.

Les exemples analysés démontrent également que la lecture citationnelle exige une construction spécifique : un infinitif accompli suivant le verbe vouloir. ${ }^{19}$ Néanmoins, il faut constater que la valeur citationnelle n'est pas forcément exclusive.

Pour ce qui est de l'analyse sémantique, nous sommes parties de l'hypothèse que la signification principale de vouloir est constituée par un certain nombre de critères. Ces critères sont prototypiques pour l'interprétation volitive du verbe modal : une force modale, un agent qui a le contrôle sur le prédicat, un événement dynamique, un décalage de temps, ainsi qu'un certain degré de probabilité. Nous avons posé la question de savoir dans quelle mesure le changement sémantique ayant abouti à l'interprétation citationnelle de vouloir pouvait se déduire de ce faisceau de facteurs.

À cette fin, nous avons regroupé les cinq facteurs dans un modèle figure/fond. Dans ce modèle, on voit que le critère de la probabilité constitue un élément qui est déjà présent dans toute interprétation volitive. Dans l'interprétation citationnelle, c'est notamment le facteur probabilité qui devient le trait distinctif. En fait, cette composante est le maillon qui lie les deux lectures. La coprésence du facteur probabilité dans les emplois volitif et citationnel nous amène à la conclusion suivante: nous avons affaire à un changement métonymique et non métaphorique. 


\section{Références primaires}

Erckmann, E. \& Chatrian, A. (1962). Histoire d'un paysan 1870. Paris : Pauvret.

Frantext. http://www.inalf.fr/frantext.htm.

Froissart. Chroniques. Ed. G. T. Diller (1972). Genève : Droz.

Giraudoux, J. (1935). La guerre de Troie n'aura pas lieu. Paris : Grasset.

Godefroy, F. (1938). Dictionnaire de l'ancienne langue française et de tous ses dialectes du IXe au XVe siècle. Paris : Librairie des Sciences et des Arts.

Guéhenno, J. (1948). Jean-Jacques : t. 1 : En marge des « Confessions » : 1712-1750. Paris : Grasset.

La Queste del Saint Graal. Ed. A. Pauphilet (1923). Paris : Champion.

L'express. Numéro 2701 du 10 avril 2003.

Martin Du Gard, R. (1913). Jean Barois. Paris: Gallimard 1962.

Sand, G. (1858). Les Beaux Messieurs de Bois-Doré. 2 tomes. Grenoble: L'Aurore 1990.

TLF (1971-1996). Trésor de la langue française. Dictionnaire de la langue du XIX au XXe siècle. (1789-1960). Paris : Gallimard.

\section{Références secondaires}

Bachtin, M. M. (1971). Probleme der Poetik Dostoevskijs. Aus dem Russischen von Adelheid Schramm. Munich : Hanser, ${ }^{2} 1963$.

Barbet, C. \& Vetters, C. (à paraître). Pour une étude diachronique du verbe modal pouvoir en français : les emplois «postmodaux ». Cahiers Cronos.

Blank, A. (1997). Prinzipien des lexikalischen Bedeutungswandels am Beispiel romanischer Sprachen. Tübingen : Niemeyer.

Bourciez, E. (1946). Eléments de linguistique romane. Quatrième édition révisée par l'auteur et par les soins de Jean Bourciez. Paris : Klincksieck.

Breslauer, Ch. (1996). Formen der Redewiedergabe im Deutschen und Italienischen. Heidelberg : Groos.

Bybee, J. L., Perkins R. D. \& Pagliuca W. (1994). The evolution of grammar. Tense, aspect and modality in the languages of the world. Chicago/Londres : University of Chicago Press.

Detges, U. (1999). Wie entsteht Grammatik? Kognitive und pragmatische Determinanten der Grammatikalisierung von Tempusmarkern. Reanalyse und Grammatikalisierung in den romanischen Sprachen. Lang, J. \& NeumannHolzschuh, I. (éd.). Tübingen : Niemeyer.

Detges, U. (2001). Grammatikalisierung. Eine kognitiv-pragmatische Theorie dargestellt am Beispiel romanischer und anderer Sprachen. Thèse d'habilitation non-publiée. Tübingen.

Detges, U., Waltereit, R. (2002). Grammaticalization vs. Reanalysis : a Semantic-Pragmatic Account of Functional Change in Grammar. Zeitschrift für Sprachwissenschaft 21.2, 151-195.

Ducrot, O. (1984). Le dire et le dit. Paris : Minuit.

Goldschmitt, S. (2007). Französische Modalverben in deontischem und epistemischem Gebrauch. Stuttgart : ibidem.

Gougenheim, G. (1929). Étude sur les périphrases verbales de la langue française. Paris : Nizet, réédité 1971.

Heine, B., Claudi, U. \& Hünnemeyer, F. (1991). Grammaticalization. A conceptual framework. Chicago/Londres : University of Chicago Press. 
Heine, B. (1995). Agent-oriented vs. Epistemic Modality - Some Observations on German Modals. Modality in Grammar and Discours. Bybee, J. L. \& Fleischman S. (éd.). Amsterdam/Philadelphia : Benjamins, 17-53.

Landvogt, A. (2007). « Discours cités ». Eine äußerungs- und erzähltheoretische Typologie der Redewiedergabe am Beispiel Gustave Flauberts. Thèse de doctorat. Würzburg.

Larreya, P. (2003). Types de modalité et types de modalisation. Aspects de la Modalité. Birkelund, M. \& Boysen, G. \& Kjaersgaard, P. S. (éd.). Tübingen : Niemeyer.

Nølke, H., Fløttum, K. \& Norén, C. (2004). ScaPoLine. La théorie de la polyphonie linguistique. Paris : Kimé.

Maingueneau, D. (1994). L'énonciation en linguistique française. Paris : Hachette, réedité 1999.

Marnette, S. (2005). Speech and Thought Presentation in French. Amsterdam/Philadelphia : Benjamins.

Palmer, F.R. (1986). Mood and Modality. Cambridge : Cambridge University Press.

Rapin, R. \& Pohl, J. (1961). A propos de vouloir, auxiliaire de futur. Le français moderne 29, 62-64.

Rosier, L. (1998). Le discours rapporté. Histoire, théories, pratiques. Bruxelles : Duculot.

Sweetser, E. (1988). Grammaticalization and semantic bleaching. Berkely Linguistic Society 14, 389-405.

Traugott, E. C. \& König, E. (1991). The semantics-pragmatics of grammaticalization. Traugott, E. C. \& Heine, B. (éds.). Approaches to grammaticalization. Vol. I : Focus on theoretical and methodical issues. Vol. I : Focus on types of grammatical markers. Amsterdam/Philadelphia : Benjamins, 189-218.

Wittgenstein, L. (1953). Philosophische Untersuchungen. Kritisch-genetische Edition. Schulte, J. (éd.). Francfort/M. Wissenschaftliche Buchgesellschaft, réédité 2001.

\footnotetext{
${ }^{1}$ L'emploi futurique de vouloir est particulièrement répandu en Suisse francophone ou dans le département de la Franche-Comté (cf. Gougenheim 1971, 89 s. ; Rapin \& Pohl 1961, 62 s.).

${ }^{2}$ Cf. Bourciez $(1946,118)$.

${ }^{3}$ Le développement d'un sens de futur d'un verbe volitif est un phénomène répandu dans différentes langues. Ainsi, Detges $(2001,147$ s.) constate que des verbes volitifs fonctionnent souvent comme 'concepts-sources' d'un marqueur futurique. Il donne des exemples de l'ancien français, de l'ancien espagnol, du romain, du tessin, du piémontais de nord, de l'anglais et de la langue créole tok pisin.
}

${ }^{4}$ Nous considérons vouloir comme auxiliaire modal puisqu'il peut exprimer deux valeurs : la volition impliquant une relation sémantique entre l'agent et l'auxiliaire, ce qui correspond à la modalité déontique, ainsi que la probabilité impliquant une relation entre le locuteur et son énoncé, ce qui correspond à la modalité épistémique.

${ }^{5}$ Nous écartons l'emploi de vouloir comme 'impératif de politesse' qui est restreint au subjonctif (p.ex. Veuillez agréer Monsieur, mes salutations distinguées) ainsi que les variantes de la tournure idiomatique cela/ça veut dire que.

${ }^{6}$ Le dernier emploi est mieux connu en allemand et anglais sous l'équivalent de 'quotatif'; suivant l'exemple de Barbet \& Vetters (à paraître) nous utiliserons par la suite le terme 'citationnel'.

${ }^{7}$ Nous conservons les sigles originaux des termes anglais : (F) force; (C) controlling agent ; (D) dynamic ; (L) later than the reference time; $(\mathrm{P})$ degree of probability.

${ }^{8} \mathrm{Ce}$ trait distingue vouloir des autres verbes modaux où la force $(\mathrm{F})$ est généralement un facteur extérieur à l'agent de contrôle (C). Cf. Goldschmitt $(2007,89)$.

${ }^{9}$ Palmer $(1986,96)$ constate également que l'événement auquel se réfèrent les emplois déontiques des auxiliaires modaux n'est pas factif. 
${ }^{10}$ Le « discours résumé » reprend une énonciation et indique le contenu du discours cité sans pour autant rendre la forme du discours originel.

${ }^{11}$ Il n'est pas exclu de sentir une faible touche volitive de la part des locuteurs cités.

${ }^{12}$ Le phénomène du dédoublement énonciatif caractéristique du discours rapporté a été décrit par Ducrot (1984) et par Maingueneau (1994). Rosier (1998) et Marnette (2005) ont spécialement étudié le discours rapporté en français.

${ }^{13}$ Cf. Nølke, Fløttum \& Norén $(2004,43)$.

${ }^{14}$ Nølke, Fløttum \& Norén $(2004,30)$.

${ }^{15}$ Ainsi Sweetser $(1990,59)$ et Bybee, Perkins \& Pagliuca $(1994,201)$ parlent d'un changement métaphorique. Cf. également Heine, Claudi \& Hünnemeyer $(1991,175)$

${ }^{16}$ Cf. Traugott \& König $(1991,209)$ ainsi que Larreya $(2003,173)$ : « La relation entre les deux types de modalité est une relation de type métonymique [...], avec, en ce qui concerne les formes, une dérivation [...] qui conduit de la modalité radicale à la modalité épistémique, et qui grammaticalise un non-dit [...]. » Même si ce changement est en effet métonymique, nous estimons qu'il ne s'agit pas d'une grammaticalisation, mais d'une réanalyse. Pour une différenciation détaillée entre grammaticalisation et réanalyse, voir Detges \& Waltereit (2002).

${ }^{17}$ Nous partons toujours du principe que le locuteur actuel est le locuteur 'primaire', alors que les locuteurs enchâssés sont secondaires. Ceci semble contredire la chronologie naturelle, puisque dans la plupart des cas, on cite des énoncés déjà proférés. Or, il existe également des rapports d'énoncés futurs, déjà plannifiés, ou bien d'énoncés non-existents, purement virtuels voire catégoriquement exclus. Pour disposer d'une description pouvant s'appliquer à tous les cas de discours rapporté, il vaut donc mieux prendre l'énonciation actuelle comme point de référence. Landvogt $(2007,86 \mathrm{~s}$.).

${ }^{18}$ Le modèle renvoie au « Doppelkreuz » du philosophe Wittgenstein $(2001,1046)$. Il s'agit d'une croix double qui peut être interprétée comme une croix blanche sur un fond noir, mais aussi comme une croix noire sur un fond blanc.

19 Pour une interprétation citationnelle, l'importance de l'infinitif accompli et non pas simple s'est avérée dans l'exemple (17). 